\title{
The LHCb Upgrade II
}

\author{
Nicola Neri*† \\ INFN Sezione di Milano, Milano, Italy \\ E-mail: nicola.neri@mi.infn.it
}

The LHCb Upgrade II will take full advantage of the capabilities of the High Luminosity LHC to search for new phenomena in flavour physics and is foreseen to take data after the long shutdown 4 of the LHC (2030). The full exploitation of the HL-LHC dataset will allow $\mathrm{LHCb}$ to perform the ultimate test of the CKM mechanism for quark mixing and $C P$ violation, and unique physics searches for phenomena beyond the Standard Model. Examples include measurements of $C P$ violating phases with ultimate precision, the measurement of the ratio $\mathscr{B}\left(B^{0} \rightarrow \mu^{+} \mu^{-}\right) / \mathscr{B}\left(B_{s}^{0} \rightarrow \mu^{+} \mu^{-}\right)$for a stringent test of supersymmetry models, studies of $b \rightarrow s \ell^{+} \ell^{-}$and $b \rightarrow d \ell^{+} \ell^{-}$transitions in both muon and electron decays, and the study of Higgs to charm quark coupling, as well as other physics opportunities that exploit the forward acceptance of the LHCb spectrometer. New detector components will improve the performance. The physics programme and the extensive ongoing $\mathrm{R} \& \mathrm{D}$ for new instrumentation are briefly summarised in this document.

The International Conference on B-Physics at Frontier Machines - BEAUTY2018

6-11 May, 2018

La Biodola, Elba Island, Italy

* Speaker.

$\dagger$ on behalf of the LHCb collaboration 


\section{Introduction}

The flavour physics community has the mandate to perform the most precise determination of the Cabibbo-Kobayashi-Maskawa (CKM) matrix that explains quark mixing and $C P$ violation in the Standard Model (SM), and to search for new phenomena through precision measurements of theoretically clean observables. The LHCb experiment has demonstrated excellent capabilities to perform precision measurements of heavy flavour hadron decays, already achieving notable results. A few representative examples are the observation of the rare $B_{s}^{0} \rightarrow \mu^{+} \mu^{-1}$ decay [1], the most precise measurement of the CKM angle $\gamma$ [2], and the discovery of the pentaquark in $\Lambda_{b}^{0} \rightarrow J / \psi p K^{-}$ decays [3]. Recent lepton flavour universality tests in $b \rightarrow s \ell^{+} \ell^{-}$transitions, in both muons and electron decays [4, 5], and in tree-level $b \rightarrow c \ell^{-} \bar{v}_{\ell}$ transitions, in both tau and muon decays [6, 7, 8], have provided intriguing results; these require further experimental investigation to assess whether they represent the first hint of physics phenomena beyond the SM or not.

The current LHCb experiment already accumulated a data sample of about $8 \mathrm{fb}^{-1}$. A major detector upgrade is planned during LS2 for the LHCb Upgrade I that is expected to accumulate $50 \mathrm{fb}^{-1}$ before 2030. Detector improvements are also planned during LS3 in particle identification, tracking and for the calorimeter.

The LHCb Upgrade II offers a unique possibility to fully exploit the High Luminosity LHC (HL-LHC) operational period, being very likely the only large-scale experiment for quark-flavour physics operating in the HL-LHC era. A more extensive discussion can be found in the Expression of Interest [9]. The experiment is being designed to allow operation up to an instantaneous luminosity of $2 \times 10^{34} \mathrm{~cm} \mathrm{~s}^{-1}$, an order of magnitude above that of Upgrade I; it is supposed to start taking data after the long shutdown 4 (LS4) in 2030, collecting a data sample corresponding to an integrated luminosity of at least $300 \mathrm{fb}^{-1}$. The istantaneous and integrated luminosity as a function of time is shown in Fig. 1.

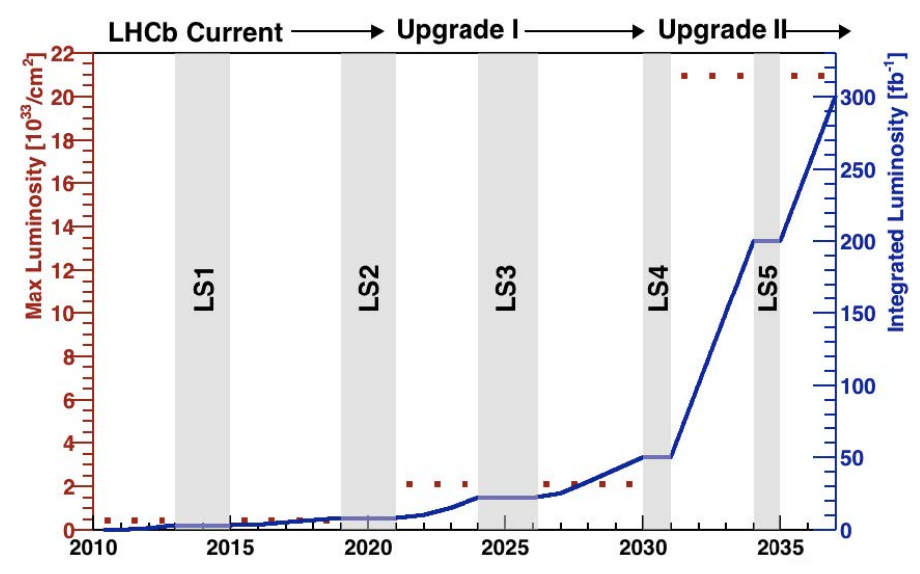

Figure 1: Luminosity projections for the LHCb, LHCb Upgrade I and LHCb Upgrade II experiments as a function of time. The red points and the left scale indicate the anticipated instantaneous luminosity during each period, with the blue line and right scale indicating the integrated luminosity accumulated.

\footnotetext{
${ }^{1}$ Charge conjugation is implied throughout the document otherwise differently stated.
} 


\section{Physics programme}

The physics programme is quite vast and it is articulated in different research areas. Beyond the standard flavour physics programme that was originally planned for the LHCb experiment, new lines of investigation emerged following the scientific interest and original proposals of the collaboration. These include the study of heavy ion collisions, and fixed-target collisions, using gas injected in the beam pipe as target. The Upgrade II programme is briefly summarised below in few main areas of interest:

- $\mathrm{CKM}$ and $C P$ violation: precision measurements of mixing and $C P$ violation in $B^{0}, B_{s}^{0}, D^{0}$, including $C P$ violation phases and ratio of CKM matrix elements, e.g. the possibility of a new measurement of $\left|V_{u b} / V_{c b}\right|$ in $B_{s}^{0} \rightarrow K^{-} \mu^{+} v_{\mu}$ decays;

- Rare decays: studies of $B_{(s)}^{0} \rightarrow \mu^{+} \mu^{-}$decays, $b \rightarrow s \ell^{+} \ell^{-}$and $b \rightarrow d \ell^{+} \ell^{-}$transitions, including both muon and electron decays for lepton universality tests, studies of semileptonic $b$-hadron decays for lepton universality tests with taus and muons in the final state;

- Spectroscopy: studies of Tetraquarks, Pentaquarks, doubly-charmed baryons, heavy baryon resonances, and other searches for new states;

- Electroweak, QCD, Exotica: studies of production and properties of $Z, W$ vector bosons, $t$ quark production, $H \rightarrow c \bar{c}$ coupling constant, searches of dark photons and long-lived particles beyond the SM;

- Ion, Fixed-target: a relatively new activity dedicated to the study of heavy ion collisions, and proton-Gas collisions in fixed-target mode, mainly devoted to the investigation of QCD and nuclear effects.

The physics programme is largely complementary to the Belle II experiment, operating at the SuperKEKB asymmetric $e^{+} e^{-}$collider and expected to collect $50 \mathrm{ab}^{-1}$ of data by 2025 . In particular for the studies involving decays with final states composed of charged tracks, LHCb will have much higher signal yields and lower backgrounds, while Belle II will have better performance for decays with neutral particles and missing energy in the final state. Other areas of the physics programme remains unique to $\mathrm{LHCb}$, notably the measurements involving $B_{s}^{0}, B_{c}^{+}$, and $b$ baryons.

\section{Selected benchmark analyses}

In the following a few selected benchmark analyses are discussed as an example of the physics reach and the discovery potential of the LHCb Upgrade II. The sensitivity predictions for Upgrade II are based on extrapolations of the present LHCb results, although a few assumptions for systematic uncertainty projections are made.

\subsection{Measurement of the CKM angle $\gamma$}

The CKM angle $\gamma$ is defined as $\gamma \equiv \arg \left[-V_{u d} V_{u b}{ }^{*} / V_{c d} V_{c b}{ }^{*}\right]$. Its measurement is extremely clean from the theoretical point of view and the remaining irreducible theory uncertainty from 

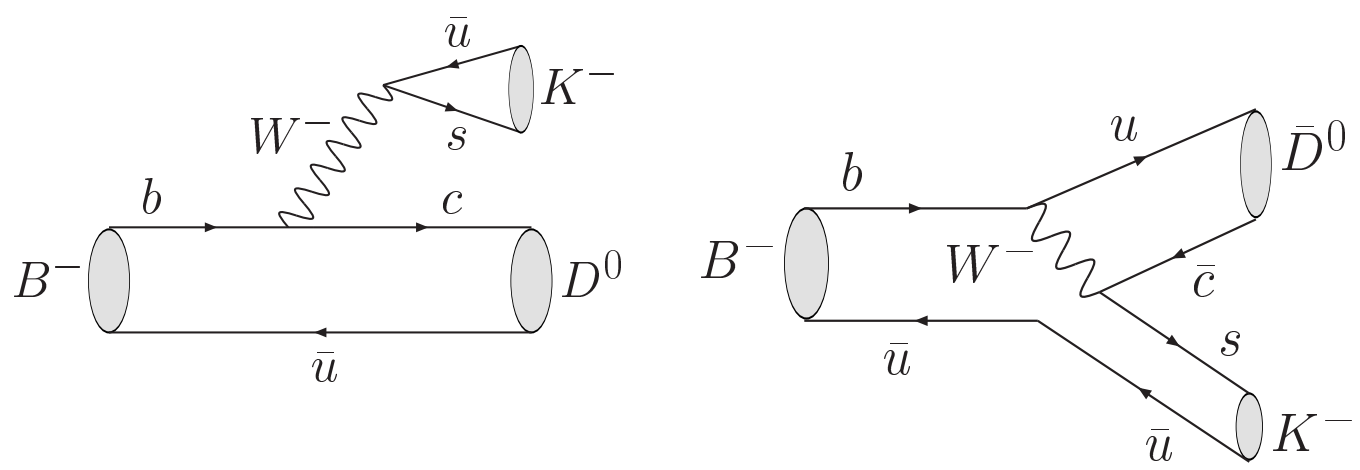

Figure 2: Leading Feynman diagrams contributing to the $B^{-} \rightarrow D K^{-}$decay. The left diagram proceeds through a $b \rightarrow c \bar{u} s$ transition $\left(\propto V_{c b} V_{u s}{ }^{*}\right)$, while the right diagram proceeds via a color-suppressed $b \rightarrow u \bar{c} s$ transition $\left(\propto V_{u b} V_{c s}{ }^{*}\right)$.

electroweak corrections is estimated to be $\delta \gamma / \gamma \simeq \mathscr{O}\left(10^{-7}\right)$ [12], which is negligibly small compared with the experimental sensitivity expected in future experiments. At present the most sensitive methods exploit the interference between the $b \rightarrow c \bar{u} s$ and $b \rightarrow u \bar{c} s$ tree-level amplitudes in $B^{-} \rightarrow D K^{-}$decays, whose leading-order diagrams are reported in Fig. 2. The sensitivity is regulated by the ratio of the interference amplitudes defined as $r_{B}^{D K}=|A(b \rightarrow u \bar{c} s) / A(b \rightarrow c \bar{u} s)| \approx 0.1$. Assuming that new physics does not affect tree-level processes, the determination of $\gamma$ is immune to effects beyond the SM, representing a reference measurement to be compared with others potentially affected by new physics. Three main methods drive the sensitivity of the measurement of the angle $\gamma$ :

- GLW method: where the $D^{0}$ is reconstructed in decay modes that are $C P$ eigenstates [13, 14];

- ADS method: with the $D^{0}$ reconstructed in doubly-Cabibbo suppressed decay modes [15, $16]$

- GGSZ method: where the $D^{0}$ is reconstructed in 3-body final states, and the analysis is based on the study of the $D^{0}$ Dalitz plot [17].

The determination of $\gamma$ is obtained from the combination of different measurements based mainly on the three methods above and several decay modes with identical quark transitions. The most recent result from LHC is $\gamma=\left(74.0_{-5.8}^{+5.0}\right)^{\circ}$ where the uncertainty includes both statistical and systematic contributions [2]. This result is consistent with the previous determinations of the BaBar and Belle experiments but has a roughly three times smaller uncertainty.

The sensitivity expected with a data sample of $300 \mathrm{fb}^{-1}$ is $0.35^{\circ}$ and is obtained through a combination of measurements. A comparison between the current LHCb GGSZ and GLW/ADS measurements with their future projections at $300 \mathrm{fb}^{-1}$ is shown in Fig. 3 (left). The projected sensitivity as a function of the integrated luminosity is shown in Fig. 3 (right) along with a comparison with the expected world average, which LHCb will dominate. Measurements of $\gamma$ from different $B^{+}, B^{0}, B_{s}^{0}$, and potentially also from $\Lambda_{b}^{0}$ decays, will allow to verify the consistency of the results and test the predictions of global CKM fits. 

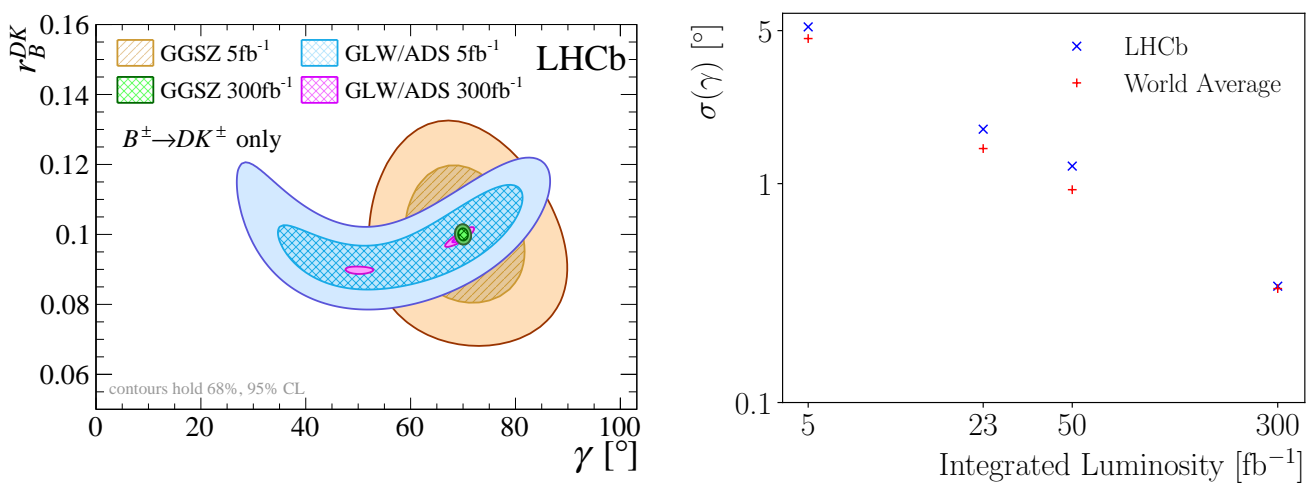

Figure 3: Left: comparison between the current LHCb 3-body GGSZ and 2-body GLW/ADS measurements alongside their future projections with $300 \mathrm{fb}^{-1}$ in the plane of $\gamma$ vs. $r_{B}^{D K}$. Right: projected sensitivity for $\gamma$ with the currently used strategies and comparison with the world average using also projections from Belle II experiment.

\section{2 $C P$ violation in neutral $B$ meson mixing}

The study of the quantum-mechanical phenomenon of flavour mixing for neutral $B$ mesons, changing particles into antiparticles during the time evolution, is sensitive to possible extensions of the SM. In particular the so-called semileptonic asymmetries,

$$
a_{s l}=\frac{\Gamma(\bar{B} \rightarrow f)-\Gamma(B \rightarrow \bar{f})}{\Gamma(\bar{B} \rightarrow f)+\Gamma(B \rightarrow \bar{f})},
$$

defined in terms of the partial decay rates, $\Gamma$, to semileptonic final states $f$, are not affected by unknown $C P$ violation in the decay, and are precisely predicted to be tiny in the SM for both $B^{0}$ and $B_{s}^{0}$ mesons. However, these values may be enhanced by non-SM contributions to the mixing process. Figure 4 shows the prospective Upgrade II measurement, arbitrarily centred at a value that differs from the SM prediction at the $10^{-3}$ level. The most relevant systematic uncertainties are represented by detection asymmetries for charged tracks with opposite charge, and background asymmetries from other $b$-hadron species. In the Upgrade II scenario, both uncertainties can be controlled using large data control samples at the $10^{-4}$ level, which is comparable with the statistical uncertainty expected with $300 \mathrm{fb}^{-1}$.

\subsection{Rare $B_{(s)}^{0} \rightarrow \mu^{+} \mu^{-}$decays}

The $B_{(s)}^{0} \rightarrow \mu^{+} \mu^{-}$decays are highly suppressed in the SM, proceeding through loop diagrams and being helicity suppressed. Their branching fractions are precisely predicted in the SM to be $\mathscr{B}\left(B_{s}^{0} \rightarrow \mu^{+} \mu^{-}\right)=(3.65 \pm 0.23) \times 10^{-9}$ and $\mathscr{B}\left(B^{0} \rightarrow \mu^{+} \mu^{-}\right)=(1.06 \pm 0.09) \times 10^{-10}$, and for these reasons these decays provide sensitive probes for physics beyond the SM. The measurements of the $B_{(s)}^{0} \rightarrow \mu^{+} \mu^{-}$effective lifetimes provide complementary information, to the measurement of the branching fraction, in the search for physics beyond the SM. The LHCb collaboration has recently measured the branching fraction $\mathscr{B}\left(B_{s}^{0} \rightarrow \mu^{+} \mu^{-}\right)=\left(3.0 \pm 0.6_{-0.2}^{+0.3}\right) \times 10^{-9}$ with a statistical significance of $7.8 \sigma$, representing the first observation of this decay in a single experiment; 


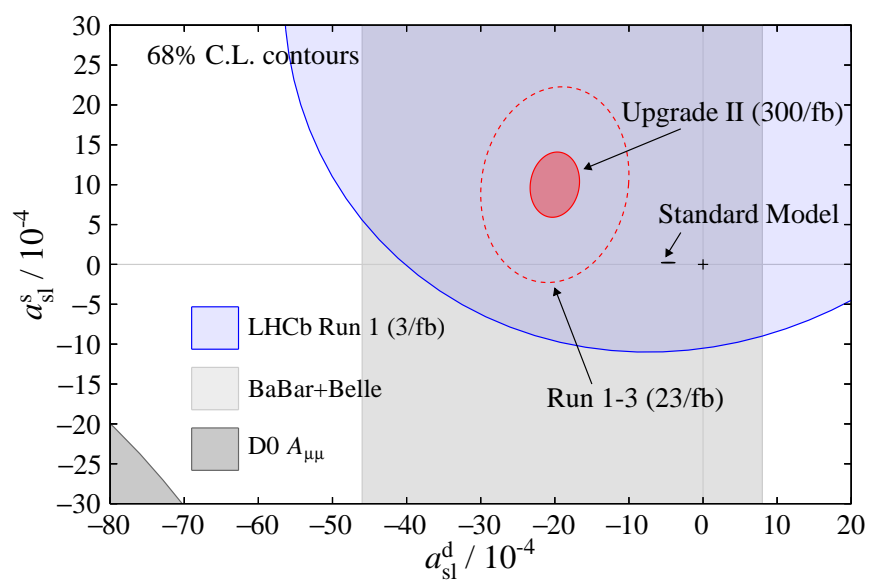

Figure 4: Current and future landscape for the semileptonic asymmetries. The grey vertical band indicates the current $B$-Factory average. The blue ellipse represents the current LHCb Run 1 measurements $[18,19]$. The red ellipse, which is arbitrarily centered, delineates the Upgrade II projected precision. The black ellipse shows the SM prediction, the uncertainty of which is barely visible with these axes.

the first measurement of the effective lifetime for the $B_{s}^{0} \rightarrow \mu^{+} \mu^{-}$decay was also performed reporting $\tau\left(B_{s}^{0} \rightarrow \mu^{+} \mu^{-}\right)=2.04 \pm 0.44 \pm 0.05$ ps. An upper limit at the $95 \%$ confidence level, $\mathscr{B}\left(B^{0} \rightarrow \mu^{+} \mu^{-}\right)<3.4 \times 10^{-10}$, was set for $B^{0} \rightarrow \mu^{+} \mu^{-}$decays where no excess of signal events was found [1].

The Upgrade II will allow the measurement of the ratio of branching fractions, $\mathscr{B}\left(B^{0} \rightarrow\right.$ $\left.\mu^{+} \mu^{-}\right) / \mathscr{B}\left(B_{s}^{0} \rightarrow \mu^{+} \mu^{-}\right)$, providing a powerful test for minimal flavour violation. The ratio can be measured with a precision of $10 \%$ using a data sample of $300 \mathrm{fb}^{-1}$, and is expected to remain limited by statistics. The projected uncertainty for the effective lifetime is about 0.03 ps [20], and would allow to discriminate between different new physics models.

\subsection{Anomalies in $b \rightarrow s \ell^{+} \ell^{-}$transitions}

Flavour-changing neutral-current decays involving $b \rightarrow s \ell^{+} \ell^{-}$transitions proceed through loop diagrams and are suppressed by the GIM mechanism in the SM. These decays are therefore promising places to search for effects of NP that could manifest themselves through deviations in angular observables, branching fractions, or violation of lepton-flavour universality. The latter refers to to the electroweak couplings of leptons that are flavour independent. The ratios of branching fractions, measured with different lepton families, is an observable that is free from hadronic uncertainties and precisely predicted. The LHCb collaboration has performed the most precise measurement of the observables $R_{K}$ and $R_{K^{*}}$ defined as

$$
R_{X}=\frac{\mathscr{B}\left(B \rightarrow X \mu^{+} \mu^{-}\right)}{\mathscr{B}\left(B \rightarrow X e^{+} e^{-}\right)} .
$$

The obtained values both show an intriguing tension with the SM expectation at the $2-2.5 \sigma$ level $[4,5]$. In the Upgrade II scenario, assuming the current detector performance, the precision 
on $R_{K}$ and $R_{K^{*}}$ will be better than $1 \%$ and could allow the discovery of physics beyond the SM, and also to distinguish among different new physics scenario, as shown in Fig. 5.

\begin{tabular}{|c|c|c|c|c|}
\hline $\begin{array}{l}\text { LHCb Upgrade II } \\
\text { Scenario-I }\end{array}$ & $\ldots$ & $\begin{array}{l}1 \\
- \\
-\end{array}$ & $\begin{array}{l}R_{K}^{\top}[1,6] \\
R_{K} \cdot[1,6] \\
R_{\phi}[1,6]\end{array}$ & $T$ \\
\hline $\begin{array}{l}\text { LHCb Upgrade II } \\
\text { Scenario-II }\end{array}$ & + & & & \\
\hline $\begin{array}{l}\text { LHCb Upgrade II } \\
\text { Scenario-III }\end{array}$ & & & & 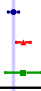 \\
\hline $\begin{array}{l}\text { LHCb Upgrade II } \\
\text { Scenario-IV }\end{array}$ & & & 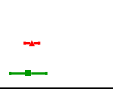 & \\
\hline LHCb Run 1 & ב & $I$ & & $\perp$ \\
\hline 0.6 & & 0.8 & & 1 \\
\hline
\end{tabular}

Figure 5: Projected sensitivity for the $R_{K}, R_{K^{*}}$ and $R_{\phi}$ measurements in different new physics scenarios with the Upgrade II data set. The existing Run 1 measurements of $R_{K}$ and $R_{K^{*}}$ are shown for comparison.

\section{Ongoing R\&D for new instrumentation}

Several detector challenges have to be overcome for being able to collect data at an instantaneous luminosity of up to $2 \times 10^{34} \mathrm{~cm}^{-2} \mathrm{~s}^{-1}$ in the forward region. About 55 visible $p p$ interactions per crossing, producing about 2500 charged particles, have to be reconstructed with the experiment fully readout at $40 \mathrm{MHz}$. Radiation-hard detectors are key to withstand fluences in excess of $10^{15} \mathrm{n}_{\text {eq }} / \mathrm{cm}^{2}$ corresponding to an integrated luminosity of $300 \mathrm{fb}^{-1}$, especially in the innermost regions of the Vertex Locator (VELO) positioned close to the interaction region. The Particle Identification (PID) detectors have to cope with high occupancy, and are requested to extend the coverage at low (around $10 \mathrm{GeV} / c$ ) and high momenta (above $100 \mathrm{GeV} / c$ ) for charged particles. The electromagnetic calorimeter (ECAL) has to sustain a dose of about $200 \mathrm{MRad}$ while maintaining a resolution similar to the current detector in Run 1 and Run 2 conditions. The Trigger and Data Acquisition (TDAQ) has to confront the biggest data processing challenge in HEP history, consisting in the acquisition and the processing in real time up to $400-500 \mathrm{~Tb} / \mathrm{s}$ of data to be reduced of about 4-5 orders of magnitude before recording to storage. Intense $R \& D$ is ongoing to overcome these detector challenges.

\subsection{Tracking with timing detectors}

The reconstruction of more than 2500 charged tracks per event in real time represents a challenge for the detector. The tracking system is designed to have increased granularity and reduced material to improve the resolution, and maintain occupancy at an acceptable level. The reduced material in the detector, especially before the dipole magnet, is also beneficial for decreasing electron bremsstrahlung in favour of their correct reconstruction. The use of fast-timing information is important for the association of heavy flavour decays to their correct primary vertex (PV) and to 
suppress ghost tracks. In Fig. 6 (left) is shown the resolution on the track impact parameter versus the inverse of the transverse momentum, $1 / p_{\mathrm{T}}$, in different scenarios of the detector and of the RF foil that separates the VELO from the primary vacuum of the LHC. Fig. 6 (right) shows the reduction of track misassociation to the PV as a function of the hit time resolution. Furthermore, the addition of fast-timing information will also bring potential improvements in track finding (see e.g. Ref. [21]). The specifications for the VELO sensors require pixel size $\lesssim 55 \mu \mathrm{m}$, hit time resolution $\lesssim 100$ ps at least for part of the layers, and excellent radiation hardness.
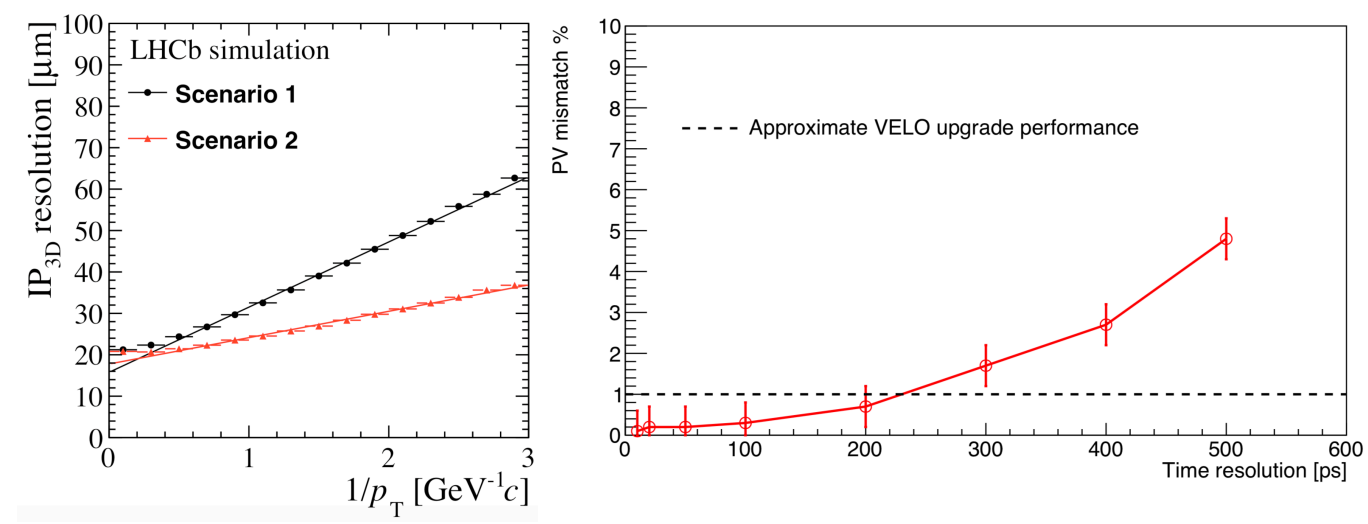

Figure 6: VELO simulated performance for Upgrade II conditions. Left: impact parameter resolution for pixels with $27 \mu \mathrm{m}$ pitch and $100 \mu \mathrm{m}$ silicon thickness (scenario 1), and also including removal of RF foil (scenario 2). Right: fraction of tracks mismatched to the wrong PV as a function of the hit time resolution.

Changes to the downstream tracking system are also foreseen to increase detector granularity in the inner part, minimise detector material and use timing information. In particular, for the inner part of the T-stations, silicon pixel sensors based on CMOS or HV-CMOS technologies of size $100 \mu \mathrm{m}$ in $x$ and $1 \mathrm{~cm}$ in $y$ directions are being considered.

\subsection{Particle identification}

High quality PID performance is crucial for precision measurements in flavour physics. Similarly to the current LHCb detector, two Ring Imaging Cherenkov detectors (RICH) will be employed: an upstream RICH 1 optimised for low momentum tracks, and a downstream RICH 2 for high momentum tracks. New photodetectors of higher granularity, e.g. $1 \mathrm{~mm}$ pitch, are under consideration, among which the SiPM technology is particularly attractive for the high quantum efficiency of about $80 \%$ at green wavelengths, offering the possibility to reduce the single-photon Cherenkov-angle uncertainty associated with the chromatic dispersion. A new optics based on flat light mirrors is being designed to reduce light aberration effects. Single photon resolution of about $0.30 \mathrm{mrad}$ and $0.13 \mathrm{mrad}$ is expected for RICH 1 and RICH 2, respectively. Around a factor of three improvement in both counters with respect to the specifications of Upgrade I.

An exciting possibility to enhance the PID performance for low-momentum tracks is represented by the TORCH detector to be installed upstream of RICH 2. The TORCH measures the time-of-flight by detecting internally reflected Cherenkov light produced in a thin $(\approx 1 \mathrm{~cm})$ quartz plane with Micro-Channel Plate (MCP) PMTs photodetectors [22]. MCP is the most promising 
technology for achieving the necessary timing resolution of 70 ps per photon to extend for kaons the particle identification into the region below $10 \mathrm{GeV}$, with an expected yield of $\approx 30$ photons per track. A proof-of-principle demonstrator was successfully tested on beam and a half-sized prototype with this application in mind will soon be constructed.

\subsection{Electromagnetic calorimeter}

The electron, photon and $\pi^{0}$ reconstruction provided by the ECAL detector is key for the physics programme at Upgrade II. The ECAL challenges are represented by the severe radiation environment, and the high number of particles per event to reconstruct at Upgrade II. The innermost modules are expected to integrate a total dose of $200 \mathrm{MRad}$, where overlapping showers would degrade the energy resolution and the shower finding efficiency. The $\pi^{0}$ meson reconstruction would suffer from large combinatoric background. To overcome these difficulties, the innermost part of the detector, corresponding to the highest occupancy region, has to be rebuilt according to a new design. The goal is to maintain at least the current detector performance, i.e. $\sigma_{E} / E=10 \% / \sqrt{E(\mathrm{GeV})} \oplus 1.5 \% \oplus 1 \% / E(\mathrm{GeV}) \sin \theta$, where $\theta$ is the polar angle of the reconstructed particle. The choice of technology has to be radiation hard, with reduced Molière radius of $2-3 \mathrm{~cm}$, and smaller size cells, e.g. $2 \times 2 \mathrm{~cm}^{2}$. A fast scintillator plus precise timing information will be essential for pile-up mitigation. Several detector technologies are being considered, including an homogeneous crystal calorimeter with longitudinal segmentation. In this respect, a promising multi-doped GAGG crystal was recently irradiated and tested in the ECAL R\&D programme. Another possibility is a sampling calorimeter, either Shashlik or SpaCal, with a tungsten-alloy converter, with a crystal component for providing a fast-timing signal. An alternative source of fast timing would be a preshower layer involving silicon pads.

\subsection{The muon system}

At Upgrade II, extra shielding is necessary to reduce the flux of pion punchtrough to a manageable level. The removal of the hadronic calorimeter, no longer required for the trigger at Upgrade II, would allow for the insertion of up to $1.7 \mathrm{~m}$ of iron, corresponding to additional 4 nuclear interactions lengths. In the innermost region, detectors with high granularity and high rate capabilities will be installed to deal with the higher particle flux . A solution is represented by the microresistive WELL detector [23], a compact spark-protected single amplification-stage Micro-Pattern Gas Detector.

\subsection{Trigger and data processing}

The Upgrade II data processing will have to deal with up to $55 \mathrm{pp}$ interactions per bunch crossing, where at most one interaction would be of interest for subsequent data analysis. Pileup suppression is key, in which detector hits not associated with the individual $p p$ interaction of interest are discarded as early as possible in the processing chain. Real-time data processing based on hybrid architectures, e.g. CPU server farms with GPU or FPGA accelerators, are being given particular attention in the ongoing $\mathrm{R} \& \mathrm{D}$, along with reconstruction algorithms tuned to take best advantage of these architectures. 


\section{Summary}

Recent $\mathrm{LHCb}$ results show some intriguing hints of new physics that require further investigation. The LHCb Upgrade II, taking data after LS4 (2030), represents a unique opportunity to take full advantage of the HL-LHC for the ultimate test of flavour physics to discover new phenomena, and with complementary discovery potential to direct searches. Other physics opportunities in the areas of spectroscopy, electroweak measurements and dark-sector searches, will complete the main programme. Many detector challenges have to be faced to build the Upgrade II detector that require a dedicated R\&D effort and also innovative solutions. LHCb Upgrade II is a well motivated, ambitious, but achievable project, with outstanding discovery potential, and a broad programme of measurements.

\section{Acknowledgments}

The author would like to thank the organisers for the invitation to such an interesting conference in a beautiful venue. Useful discussions and suggestions from LHCb colleagues are acknowledged for the preparation of this contribution, in particular from V. Gligorov, C. Parkes, and G. Wilkinson.

\section{References}

[1] LHCb Collaboration, R. Aaij et al., Measurement of the $B_{s}^{0} \rightarrow \mu^{+} \mu^{-}$branching fraction and effective lifetime and search for $B^{0} \rightarrow \mu^{+} \mu^{-}$decays, Phys. Rev. Lett. 118 (2017) 191801 LHCb-PAPER-2017-001, CERN-EP-2017-041, [1 703 . 05747].

[2] LHCB COLlaboration, Measurement of CP violation in the $B_{s}^{0} \rightarrow \phi \phi$ decay and search for the $B^{0} \rightarrow \phi \phi$ decay, May, 2018.

[3] LHCB COLlaboration , R. Aaij et al., Observation of $J / \psi$ p resonances consistent with pentaquark states in $\Lambda_{b}^{0} \rightarrow J / \psi p K^{-}$decays, Phys. Rev. Lett. 115 (2015) 072001 LHCb-PAPER-2015-029, CERN-PH-EP-2015-153, [1507.03414].

[4] LHCb COllaboration, R. Aaij et al., Test of lepton universality using $B^{+} \rightarrow K^{+} \ell^{+} \ell^{-}$decays, Phys. Rev. Lett. 113 (2014) 151601 LHCb-PAPER-2014-024, CERN-PH-EP-2014-140, [1406.6482].

[5] LHCB COllaboration, R. Aaij et al., Test of lepton universality with $B^{0} \rightarrow K^{* 0} \ell^{+} \ell^{-}$decays, JHEP 08 (2017) 055 LHCb-PAPER-2017-013, CERN-EP-2017-100, [1 705 . 05802 ].

[6] LHCb COllaboration, R. Aaij et al., Measurement of the ratio of branching fractions $\mathscr{B}\left(\bar{B}^{0} \rightarrow D^{*+} \tau^{-} \bar{v}_{\tau}\right) / \mathscr{B}\left(\bar{B}^{0} \rightarrow D^{*+} \mu^{-} \bar{v}_{\mu}\right)$, Phys. Rev. Lett. 115 (2015) 111803 LHCb-PAPER-2015-025, CERN-PH-EP-2015-150, [1506.08614].

[7] LHCB Collaboration, R. Aaij et al., Measurement of the ratio of the $\mathscr{B}\left(B^{0} \rightarrow D^{*-} \tau^{+} v_{\tau}\right)$ and $\mathscr{B}\left(B^{0} \rightarrow D^{*-} \mu^{+} v_{\mu}\right)$ branching fractions using three-prong $\tau$-lepton decays, Phys. Rev. Lett. 120 (2018) 171802 LHCb-PAPER-2017-017, CERN-EP-2017-212, [1708.08856].

[8] LHCB COLlaboration, R. Aaij et al., Test of lepton flavor universality by the measurement of the $B^{0} \rightarrow D^{*-} \tau^{+} \nu_{\tau}$ branching fraction using three-prong $\tau$ decays, Phys. Rev. D97 (2018) 072013 LHCb-PAPER-2017-027, CERN-EP-2017-256, [1711.02505]. 
[9] LHCB COLLABORATION, Expression of Interest for a Phase-II LHCb Upgrade: Opportunities in flavour physics, and beyond, in the HL-LHC era, Tech. Rep. CERN-LHCC-2017-003, CERN, Geneva, 2017.

[10] LHCB COllaboration, R. Aaij et al., Determination of the quark coupling strength $\left|V_{u b}\right|$ using baryonic decays, Nature Physics 11 (2015) 743 CERN-PH-EP-2015-084, LHCb-PAPER-2015-013, [1504.01568].

[11] LHCb COllaboration, R. Aaij et al., Observation of the doubly charmed baryon $\Xi_{c c}^{++}$, Phys. Rev. Lett. 119 (2017) 112001 LHCb-PAPER-2017-018, CERN-EP-2017-156, [1707 . 01621 ].

[12] J. Brod and J. Zupan, The ultimate theoretical error on $\gamma$ from B $\rightarrow$ DK decays, JHEP 01 (2014) 051 [1308.5663].

[13] M. Gronau and D. London, How to determine all the angles of the unitarity triangle from $B^{0} \rightarrow D K_{\mathrm{S}}^{0}$ and $B_{s}^{0} \rightarrow$ D $\phi$, Phys. Lett. B253 (1991) 483.

[14] M. Gronau and D. Wyler, On determining a weak phase from CP asymmetries in charged B decays, Phys. Lett. B265 (1991) 172.

[15] D. Atwood, I. Dunietz and A. Soni, Enhanced $C P$ violation with $B \rightarrow K D^{0}\left(\bar{D}^{0}\right)$ modes and extraction of the CKM angle $\gamma$, Phys. Rev. Lett. 78 (1997) 3257 [hep-ph/9612433].

[16] D. Atwood, I. Dunietz and A. Soni, Improved methods for observing CP violation in $B^{ \pm} \rightarrow K^{ \pm} D$ and measuring the CKM phase $\gamma$, Phys. Rev. D63 (2001) 036005 [hep-ph/ 0008090 ].

[17] A. Giri, Y. Grossman, A. Soffer and J. Zupan, Determining $\gamma$ using $B^{ \pm} \rightarrow D K^{ \pm}$with multibody D decays, Phys. Rev. D68 (2003) 054018 [hep-ph/ 0303187 ].

[18] LHCb COllaboration, R. Aaij et al., Measurement of the semileptonic CP asymmetry in $B^{0}-\bar{B}^{0}$ mixing, Phys. Rev. Lett. 114 (2015) 041601 CERN-PH-EP-2014-237, LHCb-PAPER-2014-053, [1409.8586].

[19] LHCb COllaboration, R. Aaij et al., Measurement of the CP asymmetry in $B_{s}^{0}-\bar{B}_{s}^{0}$ mixing, Phys. Rev. Lett. 117 (2016) 061803 LHCb-PAPER-2016-013, CERN-EP-2016-133, [1605. 09768 ].

[20] M. Rama, $B \rightarrow \mu^{+} \mu^{-}$and other rare decays at LHCb, PoS BEAUTY2018 (2018) 008.

[21] N. Neri, A. Cardini, R. Calabrese, M. Fiorini, E. Luppi, U. Marconi et al., 4D fast tracking for experiments at high luminosity LHC, JINST 11 (2016) C11040.

[22] M. Charles and R. Forty, TORCH: Time of flight identification with Cherenkov radiation, Nucl. Instrum. Meth. A639 (2011) 173 .

[23] G. Bencivenni, R. De Oliveira, G. Morello and M. P. Lener, The micro-Resistive WELL detector: a compact spark-protected single amplification-stage MPGD, JINST 10 (2015) P02008 [1411.2466]. 